\title{
General optimal euclidean Sobolev and Gagliardo-Nirenberg inequalities
}

\section{JURANDIR CECCON and MARCOS MONTENEGRO}

Departamento de Matemática - ICEx, Universidade Federal de Minas Gerais Av. Antônio Carlos 6627, Caixa Postal 702, 30123-970 Belo Horizonte, MG, Brasil

Manuscript received on April 5, 2005; accepted for publication on June 13, 2005; presented by JORGE HOUNIE

\begin{abstract}
We prove general optimal euclidean Sobolev and Gagliardo-Nirenberg inequalities by using mass transportation and convex analysis results. Explicit extremals and the computation of some optimal constants are also provided. In particular we extend the optimal Gagliardo-Nirenberg inequality proved by Del Pino and Dolbeault 2003 and the optimal inequalities proved by Cordero-Erausquin et al. 2004.
\end{abstract}

Key words: best constants, Gagliardo-Nirenberg inequalities, mass transportation, convex analysis.

\section{INTRODUCTION AND MAIN RESULTS}

For $1<p<n$, the optimal euclidean Sobolev inequality states that

$$
\|u\|_{p^{*}} \leq K_{n, p}\|\nabla u\|_{p}
$$

for any function $u \in \mathcal{D}^{1, p}$ (the completion of $C_{0}^{\infty}\left(\mathbb{R}^{n}\right)$ under the norm $\|\nabla u\|_{p}$ ), where

$$
\begin{gathered}
\|\nabla u\|_{p}^{p}=\int_{\mathbb{R}^{n}}|\nabla u|^{p} d x \\
p^{*}=\frac{n p}{n-p}, \quad K_{n, p}=\frac{p-1}{n-p}\left(\frac{n-p}{n(p-1)}\right)^{\frac{1}{p}}\left(\frac{\Gamma(n+1)}{\Gamma\left(\frac{n}{p}\right) \Gamma\left(n+1-\frac{n}{p}\right) \omega_{n-1}}\right)^{\frac{1}{n}} .
\end{gathered}
$$

Moreover, equality in (1) holds if and only if $u(x)=\alpha w\left(\beta\left(x-x_{0}\right)\right)$ for some $\alpha \in \mathbb{R}, \beta \neq 0$ and $x_{0} \in \mathbb{R}^{n}$, where $w(x)=\left(1+|x|^{\frac{p}{p-1}}\right)^{-\frac{n}{p^{*}}}$. This result was proved independently by Aubin 1976 and Talenti 1976.

Correspondence to: Marcos Montenegro

E-mail: montene@mat.ufmg.br 
Recently, Del Pino and Dolbeault 2003 established for $1<p<n$ the following optimal euclidean Gagliardo-Nirenberg inequality, which extends (1),

$$
\|u\|_{r} \leq \mathcal{L}_{p, q}\|\nabla u\|_{p}^{\theta}\|u\|_{q}^{1-\theta}
$$

for any function $u \in \mathcal{D}^{p, q}$ (the completion of $C_{0}^{\infty}\left(\mathbb{R}^{n}\right)$ under the norm $\|\nabla u\|_{p}+\|u\|_{q}$ ), where

$$
p<q \leq \frac{(n-1) p}{n-p}, \quad r=\frac{p(q-1)}{p-1}, \quad \theta=\frac{n(q-p)}{(n p-(n-p) q)(q-1)}
$$

and

$$
\mathcal{L}_{p, q}=\left(\frac{q-p}{p \sqrt{\pi}}\right)^{\theta}\left(\frac{p q}{n(q-p)}\right)^{\frac{\theta}{p}}\left(\frac{n p-q(n-p)}{p q}\right)^{\frac{1}{r}}\left(\frac{\Gamma\left(\frac{q(p-1)}{q-p}\right) \Gamma\left(\frac{n}{2}+1\right)}{\Gamma\left(\frac{p-1}{p} \frac{n p-q(n-p)}{q-p}\right) \Gamma\left(\frac{n(p-1)}{p}+1\right)}\right)^{\frac{\theta}{n}} .
$$

Moreover, the extremal functions of (2) are given precisely by $u(x)=\alpha w\left(\beta\left(x-x_{0}\right)\right)$ for $\alpha \in \mathbb{R}$, $\beta \neq 0$ and $x_{0} \in \mathbb{R}^{n}$, where $w(x)=\left(1+|x|^{\frac{p}{p-1}}\right)^{-\frac{p-1}{q-p}}$. Note that when $q=\frac{(n-1) p}{n-p}$, the inequality (2) becomes (1), and the same holds for the respective extremal functions.

The proofs of (1) and (2) provided in Aubin 1976, Talenti 1976 and Del Pino and Dolbeault 2003 rely on techniques from calculus of variations such as symmetrization of minimizers and uniqueness of radial solutions of Euler-Lagrange equations of related variational problems.

More recently, Cordero-Erausquim et al. 2004 introduced a new method in the proof of (1) and (2) based on a refinement by McCann 1995 of a mass transportation result due to Brenier. This new approach allowed them to generalize (1) and (2) considering arbitrary norms on $\mathbb{R}^{n}$.

The main purpose of this note is to extend (1) and (2) in the same spirit of the recent work of Gentil 2003, which presents a general version of the optimal $L^{p}$-euclidean logarithmic Sobolev inequality by using estimates of semigroups associated to Hamilton-Jacobi equations.

In order to state our results, let $C: \mathbb{R}^{n} \rightarrow \mathbb{R}$ be an even, positive $(C(x)>0, \forall x \neq 0)$, $p$-homogeneous $\left(C(\lambda x)=\lambda^{p} C(x), \forall \lambda \geq 0\right), 1<p<\infty$, and convex function. Let us denote by $C^{*}$ the Legendre transform of $C$ and by $p^{\prime}$ the conjugate of $p$, i.e. $\frac{1}{p^{\prime}}+\frac{1}{p}=1$. It is easy to show that $C^{*}$ is an even, positive, $p^{\prime}$-homogeneous and convex function.

Our main results are the following:

THEOREM 1.1 (Sobolev inequality). Let $1<p<n$ and $C$ be an even, positive, $p$-homogeneous and convex function on $\mathbb{R}^{n}$. Then for any $u \in \mathcal{D}^{1, p}$, we have

$$
\|u\|_{p^{*}} \leq K_{n, p}^{C}\|C(\nabla u)\|_{1}^{\frac{1}{p}}
$$

with $K_{n, p}^{C}=\left\|C\left(\nabla w_{C}\right)\right\|_{1}^{-\frac{1}{p}}$, where $w_{C}(x)=\left(\sigma+C^{*}(x)\right)^{-\frac{n}{p^{*}}}$ with $\sigma>0$ chosen such that $\left\|w_{C}\right\|_{p^{*}}=1$. Moreover, the extremal functions of (4) are given precisely by $u(x)=\alpha w_{C}\left(\beta\left(x-x_{0}\right)\right)$ for $\alpha \in \mathbb{R}, \beta \neq 0$ and $x_{0} \in \mathbb{R}^{n}$. 
THEOREM 1.2 (Gagliardo-Nirenberg inequality). Let $1<p<n$ and $C$ be an even, positive, $p$-homogeneous and convex function on $\mathbb{R}^{n}$. Then for any $u \in \mathcal{D}^{p, q}$, we have

$$
\|u\|_{r} \leq \mathcal{L}_{p, q}^{C}\|C(\nabla u)\|_{1}^{\frac{\theta}{p}}\|u\|_{q}^{1-\theta}
$$

with $\mathcal{L}_{p, q}^{C}=\left(\left\|C\left(\nabla w_{C}\right)\right\|_{1}^{\frac{\theta}{p}}\left\|w_{C}\right\|_{q}^{1-\theta}\right)^{-1}$, where $q, r, \theta$ satisfy (3) and $w_{C}(x)=(\sigma+$ $\left.\frac{q-p}{p-1} C^{*}(x)\right)^{-\frac{p-1}{q-p}}$ with $\sigma>0$ chosen such that $\left\|w_{C}\right\|_{r}=1$. Moreover, the extremal functions of (5) are given precisely by $u(x)=\alpha w_{C}\left(\beta\left(x-x_{0}\right)\right)$ for $\alpha \in \mathbb{R}, \beta \neq 0$ and $x_{0} \in \mathbb{R}^{n}$.

Taking $C(x)=|x|^{p}$, where $|\cdot|$ is the euclidean norm, we see that inequalities (4) and (5) extend (1) and (2), respectively. More generally, if $C(x)=\|x\|^{p}$, where $\|\cdot\|$ denotes an arbitrary norm on $\mathbb{R}^{n}$, inequalities (4) and (5) coincide with the optimal Sobolev and Gagliardo-Nirenberg inequalities established by Cordero-Erausquim et al. 2004. Note also that Theorem 1.2 provides an alternative proof of Theorem 1.1 of Gentil 2003 for $1<p<n$ and $C(x)$ only continuous by passing to the limit $q \downarrow p$ in (5).

Clearly the general conditions on $C$ do not allow us to use arguments of symmetrization and uniqueness for Euler-Lagrange equations. We instead carry out the proof of Theorems 1.1 and 1.2 by mass transportation tecniques in a similar spirit as used by Cordero-Erausquim et al. 2004, except that we shall rely on results of convex analysis involving $C$ and $C^{*}$.

REMARK 1.1. Natural generalizations of Theorems 2 and 4 of Cordero-Erausquim et al. 2004 are obtained by using a similar reasoning to that used in the proofs of Theorems 1.1 and 1.2.

As a consequence of Theorems 1.1 and 1.2, we have the explicit form of the constants $K_{n, p}^{C_{\mu}}$ and $\mathcal{L}_{p, q}^{C_{\mu}}$ for the family of $p$-homogeneous functions $C_{\mu}(x)=\frac{1}{p}|x|_{\mu}^{p}$ in terms of the optimal constants related to the classical euclidean Sobolev and Gagliardo-Nirenberg inequalities, where $|\cdot|_{\mu}$ denotes the $\mu$-norm $|x|_{\mu}=\left(\sum_{i=1}^{n}\left|x_{i}\right|^{\mu}\right)^{\frac{1}{\mu}}$ for $1 \leq \mu<\infty$ and $|x|_{\infty}=\max \left\{\left|x_{i}\right|: i=1, \ldots, n\right\}$. In fact, we have:

THEOREM 1.3. Let $1<\mu \leq \infty$ and $v$ be the conjugate of $\mu$. The optimal constants $K_{n, p}^{C_{\mu}}$ and $\mathcal{L}_{p, q}^{C_{\mu}}$ are given by

$$
\begin{gathered}
K_{n, p}^{C_{1}}=p^{\frac{1}{p}} \frac{\sqrt{\pi}}{2 \Gamma\left(\frac{n}{2}+1\right)^{\frac{1}{n}}} K_{n, p}, \quad K_{n, p}^{C_{\mu}}=p^{\frac{1}{p}}\left(\frac{\Gamma\left(\frac{1}{2}+1\right)}{\Gamma\left(\frac{1}{v}+1\right)}\right)\left(\frac{\Gamma\left(\frac{n}{v}+1\right)}{\Gamma\left(\frac{n}{2}+1\right)}\right)^{\frac{1}{n}} K_{n, p}, \\
\mathcal{L}_{p, q}^{C_{1}}=p^{\frac{\theta}{p}}\left(\frac{\sqrt{\pi}}{2 \Gamma\left(\frac{n}{2}+1\right)^{\frac{1}{n}}}\right)^{\theta} \mathcal{L}_{p, q}, \quad \mathcal{L}_{p, q}^{C_{\mu}}=p^{\frac{\theta}{p}}\left(\frac{\Gamma\left(\frac{1}{2}+1\right)}{\Gamma\left(\frac{1}{v}+1\right)}\right)^{\theta}\left(\frac{\Gamma\left(\frac{n}{v}+1\right)}{\Gamma\left(\frac{n}{2}+1\right)}\right)^{\frac{\theta}{n}} \mathcal{L}_{p, q},
\end{gathered}
$$

where $K_{n, p}$ and $\mathcal{L}_{p, q}$ are the optimal constants of (1) and (2), respectively.

Note that Theorem 1.3 expresses the optimal constants $K_{n, p}^{C_{\mu}}$ and $\mathcal{L}_{p, q}^{C_{\mu}}$ essentially in terms of $K_{n, p}^{C_{2}}$ and $\mathcal{L}_{p, q}^{C_{2}}$. Stated in this form, its proof requires several changes of variables followed by straightforward computations. Note also that the asymptotic behaviours of $K_{n, p}^{C_{\mu}}$ and $\mathcal{L}_{p, q}^{C_{\mu}}$ as $\mu$ tends to 1 are exactly $K_{n, p}^{C_{1}}$ and $\mathcal{L}_{p, q}^{C_{1}}$, respectively, as expected. 


\section{PROOF OF THEOREMS}

In the sequel we prove only Theorems 1.2 and 1.3 since the first one extends Theorem 1.1. We begin with some preliminary results. Consider functions $u, v \in C_{0}^{\infty}\left(\mathbb{R}^{n}\right)$ such that $u, v \geq 0$ and $\|u\|_{r}=\|v\|_{r}=1$. Let $\varphi$ be the Brenier map which transports the measure $u^{r}(x) d x$ onto the measure $v^{r}(x) d x$.

LEMMA 2.1. The inequality holds

$$
\frac{p(q-1)}{q-p} \int_{\mathbb{R}^{n}} v^{q} d x \leq-q \int_{\mathbb{R}^{n}} u^{q-1} \nabla u \cdot \nabla \varphi d x+\frac{p(q-1)-n(q-p)}{q-p} \int_{\mathbb{R}^{n}} u^{q} d x .
$$

ProOF. See page 322 in Cordero-Erausquim et al. 2004.

Another essential ingredient in the proofs is the following lemma:

Lemma 2.2. Let $1<p<\infty$ and $C$ be a positive, $p$-homogeneous and convex function on $\mathbb{R}^{n}$.

Then

$$
(p-1) C\left(\nabla C^{*}(x)\right)=C^{*}(x)
$$

almost everywhere.

ProOf. From the conditions under $C$ we have $C^{* *}=C$, thus $C(y)=\sup _{z \in \mathbb{R}^{n}}\left\{y \cdot z-C^{*}(z)\right\}$. The positivity and $p$-homogeneity of $C$ imply that $C^{*}$ is finite. So, from the convexity and continuity of $C^{*}$, it follows that $\nabla C^{*}(x)$ exist a.e.. Moreover, we have $\nabla C^{*}(x) \cdot x-C^{*}(x) \geq \nabla C^{*}(x) \cdot y-C^{*}(y)$ a.e. in $x$. Therefore, $C\left(\nabla C^{*}(x)\right)=\nabla C^{*}(x) \cdot x-C^{*}(x)$. On the other hand, the $p^{\prime}$-homogeneity of $C^{*}$ implies that $\nabla C^{*}(x) \cdot x=p^{\prime} C^{*}(x)$. Finally we have $C\left(\nabla C^{*}(x)\right)=\left(p^{\prime}-1\right) C^{*}(x)$ as wished.

Proof of Theorem 1.2. For $u \in \mathcal{D}^{p, q}$ we have $|u| \in \mathcal{D}^{p, q}$ and $\nabla|u|= \pm \nabla u$. So, it is sufficient to prove (5) for nonnegative functions, since $C(x)$ is an even function. Moreover, we may assume that $u \in C_{0}^{\infty}\left(\mathbb{R}^{n}\right)$ such that $\|u\|_{r}=1$ by using density and homogeneity. At first, utilizing the Fenchel inequality $x \cdot y \leq C(x)+C^{*}(y)$, with $x=-\nabla u$ and $y=u^{q-1} \nabla \varphi$, the Monge-Ampère equation $u^{r}(x)=v^{r}(\nabla \varphi(x)) \operatorname{det}\left(D^{2} \varphi(x)\right)$, satisfied by the Brenier map, and the density argument in Lemma 2.1, we find

$$
\begin{aligned}
& \frac{p(q-1)}{q(q-p)} \int_{\mathbb{R}^{n}} w_{C}^{q} d x-\int_{\mathbb{R}^{n}} C^{*}(x) w_{C}^{\frac{p(q-1)}{p-1}} d x \\
& \leq \int_{\mathbb{R}^{n}} C(\nabla u) d x+\frac{p(q-1)-n(q-p)}{q(q-p)} \int_{\mathbb{R}^{n}} u^{q} d x,
\end{aligned}
$$

where again we use that $C$ is even. Now applying this inequality to the function $u_{\lambda}(x)=$ $\lambda^{\frac{n(p-1)}{p(q-1)}} u(\lambda x)$ in the place of $u$, we obtain for any $\lambda>0$,

$$
K_{1} \leq \lambda^{\frac{n(p-q)+p(q-1)}{q-1}}\|C(\nabla u)\|_{1}+K_{2} \lambda^{\frac{n(p-q)}{p(q-1)}}\|u\|_{q}^{q}
$$

where

$$
K_{1}=\frac{p(q-1)}{q(q-p)} \int w_{C}^{q} d x-\int C^{*}(x) w_{C}^{\frac{p(q-1)}{p-1}} d x \text { and } K_{2}=\frac{p(q-1)-n(q-p)}{q(q-p)} \geq 0 .
$$


Minimizing in $\lambda$ we arrive at a general Gagliardo-Nirenberg inequality. Let us show now that $w_{C}$ is an extremal function for such general inequality. For this, it is sufficient to check that inequality (6) becomes an equality for $u=w_{C}$. In fact, equality in (6) with $u=w_{C}$ implies equality in (7) with $\lambda=1$, and therefore the infimum in (7) is achieved for $\lambda=1$ and its value is $K_{1}$. This concludes (5). The key ingredients in the proof of the equality in (6) are the following identities:

$$
\begin{aligned}
\int_{\mathbb{R}^{n}} w_{C}^{q} d x & =\frac{1}{n} \int\left(\sigma+\frac{q-p}{p-1} C^{*}(x)\right)^{-\frac{q(p-1)}{q-p}} \operatorname{div}(x) d x \\
& =\frac{q}{n} \int_{\mathbb{R}^{n}}\left(\sigma+\frac{q-p}{p-1} C^{*}(x)\right)^{-\frac{p(q-1)}{q-p}} \nabla C^{*}(x) \cdot x d x \\
& =\frac{p q}{n(p-1)} \int_{\mathbb{R}^{n}}\left(\sigma+\frac{q-p}{p-1} C^{*}(x)\right)^{-\frac{p(q-1)}{q-p}} C^{*}(x) d x \\
& =\frac{p q}{n(p-1)} \int_{\mathbb{R}^{n}} C^{*}(x) w_{C}^{\frac{p(q-1)}{p-1}} d x,
\end{aligned}
$$

where we utilize integration by parts and the property $\nabla C^{*}(x) \cdot x=\frac{p}{p-1} C^{*}(x)$, and

$$
\begin{gathered}
\int_{\mathbb{R}^{n}} C\left(\nabla\left(w_{C}\right)\right) d x=\int_{\mathbb{R}^{n}} C\left(\left(\sigma+\frac{q-p}{p-1} C^{*}(x)\right)^{-\frac{q-1}{q-p}} \nabla C^{*}(x)\right) d x \\
=\frac{1}{p-1} \int_{\mathbb{R}^{n}}\left(\sigma+\frac{q-p}{p-1} C^{*}(x)\right)^{-\frac{p(q-1)}{q-p}} C^{*}(x) d x=\frac{1}{p-1} \int_{\mathbb{R}^{n}} C^{*}(x) w_{C}^{\frac{p(q-1)}{p-1}} d x,
\end{gathered}
$$

by Lemma 2.2. Replacing these relations in (6), the desired equality follows. Finally, let $u$ be an extremal for (5). Adaptating the proof of Theorem 5 in Cordero-Erausquim et al. 2004, one concludes that the inequality stated in Lemma 2.1 becomes an equality with $v=w_{C}$. Next, proceeding as in Cordero-Erausquim et al. 2004 we finish the proof.

Proof OF THEOREM 1.3. In the sequel, we only furnish the steps of the calculus of $\mathcal{L}_{p, q}^{C_{\mu}}$. The form of the optimal Sobolev constant follows by taking $q=\frac{(n-1) p}{n-p}$. Lemma 2.2 applied to $C^{*}$ in the place of $C$ furnishes $C^{*}(\nabla C(x))=(p-1) C(x)$. Using this relation with $C=C_{\mu}$, we find $C_{\mu}^{*}(x)=\frac{1}{p^{\prime}}|x|_{\nu}^{p^{\prime}}$ for $1<\mu<\infty$. By approximation, we also have $C_{1}^{*}(x)=\frac{1}{p^{\prime}}|x|_{\infty}^{p^{\prime}}$ and $C_{\infty}^{*}(x)=\frac{1}{p^{\prime}}|x|_{1}^{p^{\prime}}$. The computation of $\mathcal{L}_{p, q}^{C_{\mu}}$ depends on the integral

$$
\int_{S^{n-1}} \prod_{i=1}^{n}\left|y_{i}\right|^{\frac{2-v}{v}} d s
$$

where $S^{n-1}$ denotes the $(n-1)$-dimensional unit sphere. In order to compute this integral we consider the chart $\psi: D^{n-1} \subset \mathbb{R}^{n-1} \rightarrow S^{n-1}$ defined by $\psi\left(y_{1}, \cdots, y_{n}\right)=\left(y_{1}, \cdots, y_{n-1},(1-\right.$ $\left.\left.\left(y_{1}^{2}+\cdots+y_{n-1}^{2}\right)\right)^{\frac{1}{2}}\right)$, where $D^{n-1}$ denotes the unit ball in $\mathbb{R}^{n-1}$. Clearly, the Riemmanian metric 
$g$ of $S^{n-1}$ on the chart $\psi$ satisfies $|g|=\frac{1}{1-\left(y_{1}^{2}+\cdots+y_{n-1}^{2}\right)}$. So, we have

$$
\begin{aligned}
\int_{S^{n-1}} \prod_{i=1}^{n}\left|y_{i}\right|^{\frac{2-v}{v}} d s & =2 \int_{D^{n-1}} \frac{\left|y_{1}\right|^{\frac{2-v}{v}} \cdot \cdots \cdot\left|y_{n-1}\right|^{\frac{2-v}{v}}\left(1-\left(y_{1}^{2}+\cdots+y_{n-1}^{2}\right)^{\frac{2-v}{2 v}}\right.}{\left(1-\left(y_{1}^{2}+\cdots+y_{n-1}^{2}\right)\right)^{\frac{1}{2}}} d y \\
& =2 \int_{D^{n-1}}\left|y_{1}\right|^{\frac{2-v}{2}} \cdots \cdot\left|y_{n-1}\right|^{\frac{2-v}{v}}\left(1-\left(y_{1}^{2}+\cdots+y_{n-1}^{2}\right)\right)^{\frac{1-v}{v}} d y \\
& =2 \int_{0}^{1} \int_{S^{n-2}}\left|y_{1}\right|^{\frac{2-v}{v}} \cdots \cdot\left|y_{n-1}\right|^{\frac{2-v}{v}}\left(1-r^{2}\right)^{\frac{1-v}{v}} r^{\frac{(2-v)(n-1)}{v}+n-2} d s d r \\
& =2 \int_{0}^{1}\left(1-r^{2}\right)^{\frac{1-v}{v}} r^{\frac{2(n-1)}{v}}-1 d r \int_{S^{n-2}} \prod_{i=1}^{n-1}\left|y_{i}\right|^{\frac{2-v}{v}} d s .
\end{aligned}
$$

By finite induction on $n$, it follows immediately that

$$
\int_{S^{n-1}} \prod_{i=1}^{n}\left|y_{i}\right|^{\frac{2-v}{v}} d s=2^{n} \prod_{i=1}^{n-1} \int_{0}^{1}\left(1-r^{2}\right)^{\frac{1-v}{v}} r^{\frac{2(n-i)}{v}-1} d r .
$$

Now, using some properties of the gamma function, we find

$$
\int_{S^{n-1}} \prod_{i=1}^{n}\left|y_{i}\right|^{\frac{2-v}{v}} d s=\frac{2 n \Gamma\left(\frac{1}{v}\right)^{n}}{v \Gamma\left(\frac{n}{v}+1\right)} .
$$

According to Theorem 1.2, the function

$$
w(x)=\left(1+C_{\mu}^{*}(x)\right)^{-\frac{p-1}{q-p}}=\left(1+\frac{1}{p^{\prime}}|x|_{\nu}^{p^{\prime}}\right)^{\frac{p-1}{p-q}}
$$

is extremal for $\mathcal{L}_{p, q}^{C_{\mu}}$. On the other hand, using the change of variable $x_{i}=\left(\frac{1}{p^{\prime}}\right)^{\frac{1}{p^{\prime}}}\left|y_{i}\right|^{\frac{2-v}{v}} y_{i}$ followed by straightforward computations, we obtain

$$
\begin{aligned}
& \left\|C_{\mu}(\nabla w)\right\|_{1}=\frac{1}{p} p^{\prime \frac{n}{p^{\prime}}}\left(\frac{2}{v}\right)^{n} \frac{v}{2}\left(\frac{p-1}{q-p}\right)^{p} \int_{0}^{\infty} \frac{t^{\frac{n}{p^{\prime}}}}{(1+t)^{\frac{p(q-1)}{q-p}}} d t \int_{S^{n-1}} \prod_{i=1}^{n}\left|y_{i}\right|^{\frac{2-v}{v}} d s, \\
& \|w\|_{q}^{q}=p^{\prime \frac{n}{p^{\prime}}}\left(\frac{2}{v}\right)^{n} \frac{v}{2 p^{\prime}} \int_{0}^{\infty} \frac{t^{\frac{n}{p^{\prime}}-1}}{(1+t)^{\frac{q(p-1)}{q-p}}} d t \int_{S^{n-1}} \prod_{i=1}^{n}\left|y_{i}\right|^{\frac{2-v}{v}} d s
\end{aligned}
$$

and

$$
\|w\|_{r}^{r}=p^{\prime} \frac{n}{p^{\prime}}\left(\frac{2}{v}\right)^{n} \frac{v}{2 p^{\prime}} \int_{0}^{\infty} \frac{t^{\frac{n}{p^{\prime}}-1}}{(1+t)^{\frac{p(q-1)}{q-p}}} d t \int_{S^{n-1}} \prod_{i=1}^{n}\left|y_{i}\right|^{\frac{2-v}{v}} d s .
$$

Finally, replacing (9) in these relations and using some properties of the gamma function, we arrive at the desired form of the constant $\mathcal{L}_{p, q}^{C_{\mu}}$.

\section{ACKNOWLEDGMENTS}

The first author was partially supported by Conselho Nacional de Desenvolvimento Científico e Tecnológico (CNPq). 


\section{RESUMO}

Provamos desigualdades ótimas euclideanas de Sobolev e de Gagliardo-Nirenberg gerais via método de transporte de massa e resultados de análise convexa. Obtemos funções extremais explícitas e calculamos algumas melhores constantes. Em particular, estendemos a desigualdade ótima de Gagliardo-Nirenberg obtida por Del Pino e Dolbeault 2003 e as desigualdades ótimas obtidas por Cordero-Erausquin et al. 2004.

Palavras-chave: melhores constantes, desigualdades de Gagliardo-Nirenberg, transporte de massa, análise convexa.

\section{REFERENCES}

Aubin T. 1976. Problèmes isopérimétriques et espaces de Sobolev. J Diff Geom 11: 573-598.

Cordero-Erausquin D, NAZARet B AND Villani C. 2004. A mass-transportation approach to sharp Sobolev and Gagliardo-Nirenberg inequalities. Adv Math 182: 307-332.

Del Pino M and Dolbeault J. 2003. The optimal euclidean $L^{p}$-Sobolev logarithmic inequality. J Funct Anal 197: 151-161.

GeNTIL I. 2003. The general optimal $L^{p}$-euclidean logarithmic Sobolev inequality by Hamilton-Jacobi equations. J Funct Anal 202: 591-599.

MCCANN RJ. 1995. Existence and uniqueness of monotone measure-preserving maps. Duke Math J 80: 309-323.

TALenti G. 1976. Best constant in Sobolev inequality. Ann Mat Pura Appl (iv) 110: 353-372. 